\title{
Resveratrol Protects Neurons and the Myocardium by Reducing Oxidative Stress and Ameliorating Mitochondria Damage in a Cerebral Ischemia Rat Model
}

\author{
Ran Wang Yan-yan Liu Xiao-yu Liu Shu-wei Jia Jian Zhao Dan Cui Ling Wang \\ Department of Physiology, Harbin Medical University, Harbin, People's Republic of China
}

\section{Key Words}

Resveratrol • Cerebral ischemia • Mitochondria • Oxidative stress • Apoptosis • Autophagy

\begin{abstract}
Background: Resveratrol has shown potent antioxidant activity in ischemia models. The present study was performed to determine whether resveratrol protects against cerebral ischemiainduced neuronal and myocardial injury by interfering with mitochondrial homeostasis. Methods: Wistar rats were pretreated with resveratrol or vehicle intraperitoneally for one week and then subjected to cerebral ischemia via middle cerebral artery occlusion (MCAO) for $24 \mathrm{~h}$. Oxidation was evaluated by quantitating SOD activity and MDA levels. Apoptosis and autophagy were measured based on TUNEL staining and the expression levels of $\mathrm{Bcl}-2$, Bax and LC3II. Mitochondrial changes were evaluated by transmission electron microscopy and by analyzing the mitochondrial membrane potential. Results: Resveratrol significantly decreased mortality, neurological deficits, infarction volume and MDA levels and increased SOD activity. Furthermore, neurocyte apoptosis was alleviated by resveratrol as indicated by the increased $\mathrm{BCl}-2 / \mathrm{Bax}$ ratio, increased LC3II expression and a decreased number of TUNELpositive neurocytes. Resveratrol preserved the mitochondria in neurons and cardiomyocytes and significantly improved cardiac function. Conclusion: Resveratrol protected brain tissues against ischemic damage by interfering with mitochondrial homeostasis and inhibiting apoptosis. Furthermore, resveratrol attenuated myocardial damage, suggesting that it may be a novel therapy for cerebral ischemia diseases.
\end{abstract}




\section{Cellular Physiology and Biochemistry}

Cell Physiol Biochem 2014;34:854-864

DOI: $10.1159 / 000366304$

C) 2014 S. Karger AG, Basel

www.karger.com/cpb

Wang et al.: Protective Roles of Resveratrol in Cerebral Ischemia

\section{Introduction}

Ischemic cerebrovascular disease leads to approximately 6 million deaths every year worldwide [1]. In addition to neurological damage, other important causes of mortality from ischemic cerebrovascular disease are cardiac dysfunction and myocardial damage during the initial period of cerebral ischemia [2,3]. Tremendous progress has been made in understanding the pathophysiology of strokes; however, effective therapies are still lacking for stroke patients, and the approved stroke therapy is limited by its narrow time window for application [4,5]. Recently, antioxidant treatments have begun to be investigated as potential stroke therapeutics. Increasing evidence has demonstrated that reactive oxygen species (ROS) contribute to ischemic injury. Although the sources for and mechanisms of ROS generation during ischemia remain elusive, evidence from studies using antioxidants or ROS-overproduction approaches have demonstrated the central roles of ROS in cell death signaling pathways $[6,7]$ and have determined that cell death plays a critical role in ischemic injury.

Apoptosis and autophagy are two major pathways of cell death. Apoptosis is regulated by Bcl-2 family proteins $[8,9]$. The delayed neuronal death caused by ischemia has been attributed to apoptosis, which has been intensively studied. Recently, autophagy has also been determined to be crucial in the process of cellular apoptosis. Autophagy is an active response to various stresses in the body that eliminates damaged structures [10]. Autophagy prevents cells from undergoing apoptosis by degrading damaged mitochondria [11]. Resveratrol has been associated with increased autophagy in certain diseases [12,13].

Resveratrol (3,4,5-trihydroxy-trans-stilbene) is a unique antioxidant and antiinflammatory compound and is the key element in red wine that accounts for the "French Paradox"[14]. Extensive research has revealed that resveratrol protects against stroke and brain trauma in animal models and is being tested in clinical trials $[15,16]$. Numerous studies have revealed that mitochondria are central to the pathogenesis of stroke and other neurodegenerative diseases $[17,18]$. Previous studies have demonstrated that resveratrol treatment exerts beneficial protective effects regarding mitochondrial function in the heart and brain $[19,20]$. However, the molecular mechanisms underlying the neuroprotective effects of resveratrol are not fully understood.

The protective mechanism of action of resveratrol in rats with cerebral ischemia, especially when accompanied by myocardial damage, remains poorly defined. In this study, we aimed to determine whether resveratrol administration before MCAO could reduce ischemia-induced brain and myocardial injuries by regulating apoptosis and autophagy.

\section{Materials and Methods}

Animals and Experimental Protocols

Healthy male Wistar rats (3-4 months old, $250 \pm 20 \mathrm{~g}$ ) were used in this study. All the rats were housed at the animal facility with a room temperature of $23 \pm 1^{\circ} \mathrm{C}, 55 \pm 5 \%$ humidity, and a $12 \mathrm{~h}$ dark/light cycle and were allowed unlimited food and water. The animals were fasted overnight before the experiment. All the experimental protocols were pre-approved by the Experimental Animal Ethic Committee of Harbin Medical University, China.

The rats were randomly divided into four groups: sham operation (Sham, $\mathrm{n}=70)$, MCAO (MCAO, $\mathrm{n}=$ 90), resveratrol-treated MCAO (Res, $n=90$ ) and vehicle-treated MCAO (Veh, $n=90)$. Resveratrol (SigmaAldrich) was freshly prepared in 50\% ethanol [15]. The rats were intraperitoneally injected with $30 \mathrm{mg} / \mathrm{kg}$ resveratrol once a day for 7 days before surgery and $30 \mathrm{~min}$ before inducing ischemia, with the volume not exceeding $0.1 \mathrm{ml} / 100 \mathrm{~g}$ body weight. The resveratrol dose and the treatment protocol were selected based on previous reports that demonstrated the antioxidant property of resveratrol in different experimental models at doses ranging from $8-40 \mathrm{mg} / \mathrm{kg}[21,22]$. 


\section{Cellular Physiology and Biochemistry}

Cell Physiol Biochem 2014;34:854-864

DOI: $10.1159 / 000366304$

Publisned online: August 21, 2014

Wang et al.: Protective Roles of Resveratrol in Cerebral Ischemia

Cerebral Ischemia Induced by Middle Cerebral Artery Occlusion

The operating procedure for occluding the middle cerebral artery has been previously described [23]. Briefly, the rats were anesthetized with $10 \%$ chloral hydrate $(300 \mathrm{mg} / \mathrm{kg}$, ip). After a midline incision in the neck, the right external carotid artery was carefully exposed and dissected. A paraffin wax-coated fishing thread (diameter $0.28 \mathrm{~mm}$ ) was inserted from the external carotid artery to the right internal carotid artery $(17.5 \pm 0.5 \mathrm{~mm})$ to occlude the origin of the right middle cerebral artery (MCA) for $24 \mathrm{~h}$. In sham-operated rats, the thread was removed as soon as the origin of the MCA was reached.

After $24 \mathrm{~h}$, the neurological deficit was independently assessed by two observers who were blinded to the operating procedures based on a 5-point neuroscore $(0=$ no visible neurological deficits, $1=$ forelimb flexion, 2 = weak contralateral forelimb grip, 3 = circling to the paretic side only when pulled by the tail, and 4 = unable to walk spontaneously). All further experimental procedures were blinded by pseudonymization.

\section{Measurements of Hemodynamic Function of the heart}

Hemodynamic function was assessed $24 \mathrm{~h}$ after the MCAO operation. The left ventricular end diastolic pressure (LVEDP), left ventricular systolic pressure (LVSP), and time derivatives of the pressure change were measured during contraction $(+\mathrm{dP} / \mathrm{dt})$ and relaxation $(-\mathrm{dP} / \mathrm{dt})$ using a polygraph as described previously [24].

\section{Measurement of Cerebral Infarct Volume}

Rats were re-anesthetized and sacrificed $24 \mathrm{~h}$ after operation. The brains were removed quickly and cut into $2 \mathrm{~mm}$ thick coronal slices. The infarct volume was determined by TTC staining as previously described $[25,26]$. The brain slices were evaluated by an investigator who was blinded to the treatment. The infarct and total hemispheric areas of each section were traced and analyzed using Image-Pro Plus Data Analysis Program (Media Cybernetics, Silver Spring, MD, USA). The infarct ratio was calculated by dividing the infarct volume by the total volume of the sections.

\section{Detection of SOD and MDA in the Hippocampus}

Ischemic hippocampi were collected $24 \mathrm{~h}$ after MCAO. SOD activity and MDA levels were measured using assay kits (Nanjing Jiancheng Biotechnology Co., Nanjing, China) according to the manufacturers' instructions. The brains were rinsed, weighed, and homogenized, and then $0.5 \mathrm{ml}$ of tissue homogenate was added to a test tube and heated in boiling water for $40 \mathrm{~min}$. The solution was then cooled, and the absorbance was measured using a spectrophotometer (UV-WFZ75, Shanghai, China).

\section{TUNEL Staining for Neuronal Apoptosis in the Hippocampus}

The paraffin-embedded sections were analyzed by TUNEL staining. An in situ cell death detection kit was used to detect apoptotic cells. As directed by the product specifications, the sections were deparaffinized and rehydrated by heating at $60{ }^{\circ} \mathrm{C}$ for $10 \mathrm{~min}$. These sections were incubated in proteinase $\mathrm{K}(20 \mu \mathrm{g} / \mathrm{ml})$ for $15 \mathrm{~min}$ and then quenched for $10 \mathrm{~min}$ in 3\% hydrogen peroxide at room temperature. After three 10 min washes in PBS, the sections were incubated with TUNEL reaction mixture for $1 \mathrm{~h}$ at $37^{\circ} \mathrm{C}$. The sections were washed in PBS three times for $10 \mathrm{~min}$ each, and then color development was performed in the dark with DAB, which was used as a nuclear counter stain. The number of TUNEL-positive CA1 neurons per mm length of the medial CA1 pyramidal cell layer was carefully counted in three sections per animal. The hippocampal cell counts for each of the three sections were averaged to obtain the mean value.

\section{Western Blotting}

Total protein samples were extracted from the hippocampus using previously described procedures [27]. The tissues were lysed in RIPA lysis buffer, and $60 \mu \mathrm{g}$ of protein was separated on a $15 \%$ SDSpolyacrylamide gel. After electrophoretically transferring the proteins to a PVDF membrane, the membranes were blocked with 5\% BSA in Tris-buffered saline (TBS) for $1 \mathrm{~h}$ at room temperature. Each piece of membrane was then separately incubated with a rabbit polyclonal anti-Bax (Cell Signaling Technology, MA, USA), antibcl-2 (Santa Cruz, CA, USA) or anti-LC3B (Sigma-Aldrich) antibody overnight at $4{ }^{\circ} \mathrm{C}$ in TBST, with $\beta$-actin as the internal control (Santa Cruz, CA, USA). The Western blots were imaged on an Odyssey Infrared Imaging System (LI-COR Biosciences), and the bands were quantified using Odyssey v1.2 software by measuring the band intensity (area $\times$ OD) in each group and normalizing the intensity to the internal control. 


\section{Cellular Physiology and Biochemistry}

Cell Physiol Biochem 2014;34:854-864

DOI: $10.1159 / 000366304$

C) 2014 S. Karger AG, Basel

www.karger.com/cpb

Wang et al.: Protective Roles of Resveratrol in Cerebral Ischemia

Transmission Electron Microscopy

A piece of hippocampal tissue and a piece of left ventricular muscle from the cardiac apex $(1 \mathrm{~mm} \times 1$ $\mathrm{mm} \times 1 \mathrm{~mm}$ ) were immediately removed, fixed in $4 \%$ glutaraldehyde for $48 \mathrm{~h}$, and postfixed in $1 \%$ osmium tetroxide for $1 \mathrm{~h}$. The blocks were then dehydrated in an ethanol series, and slices were cut with an ultrathin microtome. The slices were observed using a JEM-2010 transmission electron microscope (JEOL Ltd, Tokyo, Japan).

\section{Mitochondrial Membrane Potential in Neurons and Cardiomyocytes}

The mitochondrial membrane potential $(\Delta \psi \mathrm{m})$ was measured using a JC-1 assay kit (Beyotime Institute of Biotechnology, China) according to the manufacturers' instructions. Briefly, mitochondria were isolated from fresh hippocampal tissue and left ventricular muscle using a tissue mitochondrial isolation kit (Beyotime, China). Next, $0.1 \mathrm{ml}$ of the isolated mitochondrial protein $(40 \mu \mathrm{g})$ was incubated with $0.9 \mathrm{ml}$ JC-1 staining solution. After $10 \mathrm{~min}$, the absorbance was measured with a fluorescence spectrophotometer (Varian Australia Pty Ltd, Melbourne, Victoria, Australia), and the fluorescent intensity of mitochondrial JC-1 monomers $\left(\lambda_{\text {ex }} 490 \mathrm{~nm}, \lambda_{\text {em }} 530 \mathrm{~nm}\right)$ and aggregates $\left(\lambda_{\mathrm{ex}} 525 \mathrm{~nm}, \lambda_{\mathrm{em}} 590 \mathrm{~nm}\right)$ was determined. The $\Delta \psi \mathrm{m}$ in each treatment group was calculated as the ratio of aggregates to monomers.

\section{LDH and CK Release Assay}

At $24 \mathrm{~h}$ post-MCAO, blood samples were collected from the ventral aorta and were centrifuged at $3000 \mathrm{~g}$ for $10 \mathrm{~min}$ to isolate the serum. Myocardial cellular damage was evaluated by measuring lactate dehydrogenase (LDH) and creatinine kinase (CK) activity in plasma using commercially available assay kits (Nanjing Jiancheng Biotechnology Co., Nanjing, China).

\section{Data Analysis and Statistics}

All the data are presented as the mean \pm SEM. One-way analysis of variance (ANOVA) followed by the Turkey test was used for multiple comparisons. A two-tailed value of $p<0.05$ was considered to be statistically significant. The data were analyzed using GraphPad Prism 5.0 and SPSS 13.0.

\section{Results}

Resveratrol exhibited a critical protective effect against cerebral ischemic damage in vivo

To determine whether resveratrol protected against cerebral ischemic damage in vivo, we performed survival experiments as an independent preliminary experiment. The mortality of MCAO rats was significantly attenuated by resveratrol pretreatment (log-rank test, $\mathrm{p}=0.0474$ ) (Fig. 1). Rats died as early as $1 \mathrm{~h}$ after MCA0, whereas the resveratroltreated rats started dying at $5 \mathrm{~h}$. In the MCAO group, the death rate was 20\% (4/20) within $6 \mathrm{~h}, 35 \%$ (7/20) within $24 \mathrm{~h}$ and 45\% (9/20) within $48 \mathrm{~h}$ after MCAO. In the resveratrol pretreatment group, the death rate was $5 \%(1 / 20)$ within $6 \mathrm{~h}, 15 \%(3 / 20)$ within $24 \mathrm{~h}$ and $15 \%(3 / 20)$ within $48 \mathrm{~h}$ after MCAO. These results indicated that resveratrol was essential for prolonging survival after MCAO.

\section{Neuroprotective effect of resveratrol against ischemia-induced brain injury}

After rats were subjected to $24 \mathrm{~h}$ of cerebral ischemia in the stroke model, the neurological scores and cerebral infarct size were evaluated (Fig. 2). Animals in the MCAO group displayed characteristics of ischemic injury, with higher neurological deficit scores than the resveratrol-treated rats. The average neurological score in the MCAO group was significantly decreased by resveratrol, from $2.56 \pm 1.09$ to $1.52 \pm 0.97(\mathrm{P}=0.004)$ (Fig. 2A). The cerebral infarct volume in the MCAO group was also significantly decreased by resveratrol, from $25.13 \pm 8.23 \%$ to $15.84 \pm 5.21 \%$ ( $\mathrm{P}=0.008$ ) (Fig. 2B). Figure $2 \mathrm{C}$ shows the typical morphological characteristics of the brain sections from each group after $2 \%$ TTC staining. TTC staining revealed an extensive pale infarct area in the brain sections from rats in the MCAO group, whereas there was no infarct area in the sham-treated animals. Resveratrol pretreatment significantly decreased the infarct area. These data indicated that resveratrol exerted significant neuroprotection against ischemia-induced brain injury. 
Fig. 1. Effect of resveratrol on survival after MCAO. Kaplan-Meier survival curve. Death after MCAO was significantly attenuated by resveratrol pretreatment ( $\mathrm{P}<0.05$ vs. MCAO, 95\% confidence interval, logrank test; $\mathrm{n}=20$ rats/group).
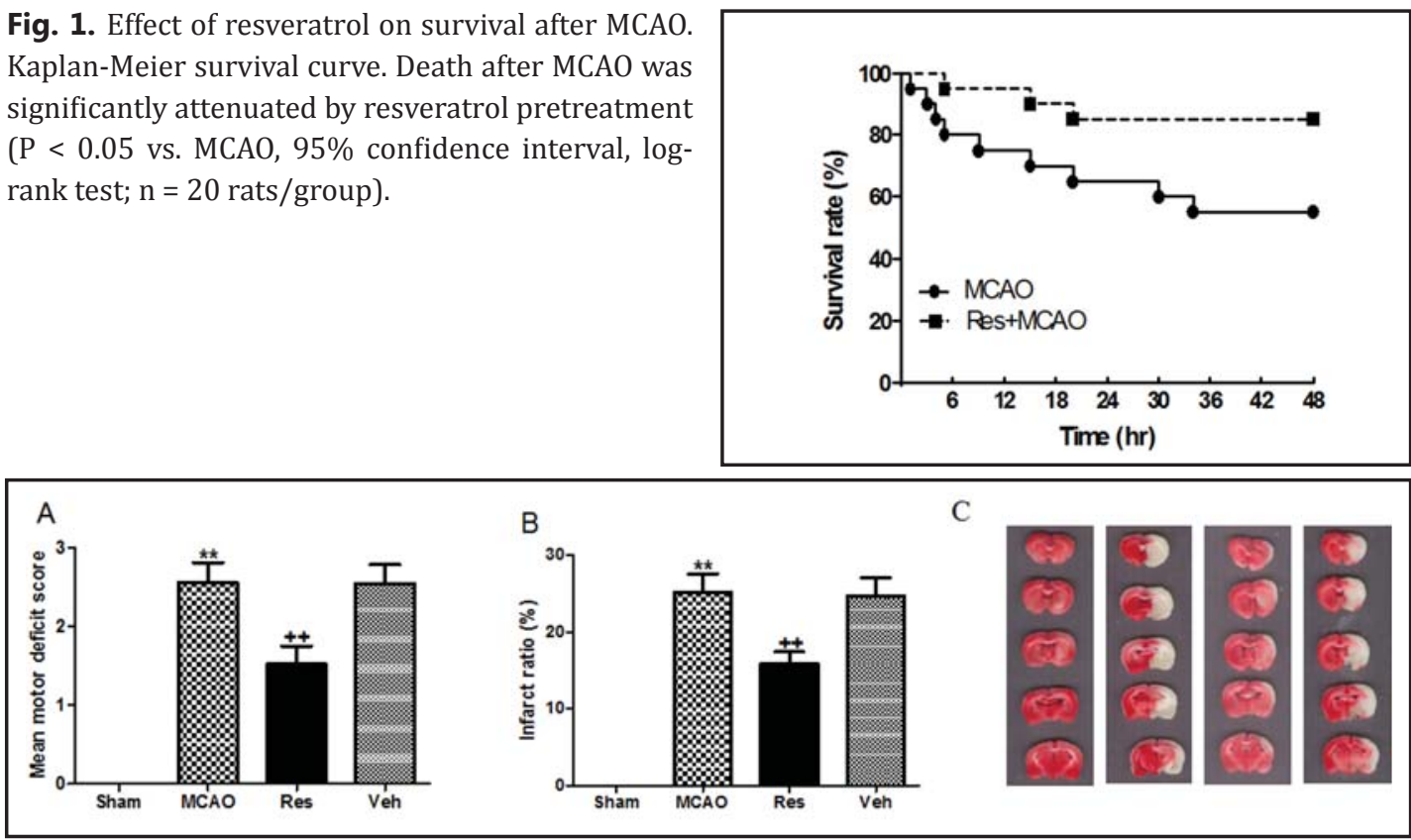

Fig. 2. Effect of resveratrol on neurological deficits and infarct size after $24 \mathrm{~h}$ of MCAO. (A) Neurological scores significantly decreased after MCAO, and resveratrol pretreatment improved neurological function compared with the MCAO group. (B) Resveratrol decreased the infarct area in rats with an MCAO. (C) Coronal sections from ischemic rat brains stained with TTC. Red represents normal tissue. White represents infarct tissue. ( ${ }^{* *} \mathrm{P}<0.01$ vs. Sham; $++\mathrm{P}<0.01$ vs. MCAO; $\mathrm{n}=10$ rats/group).

A

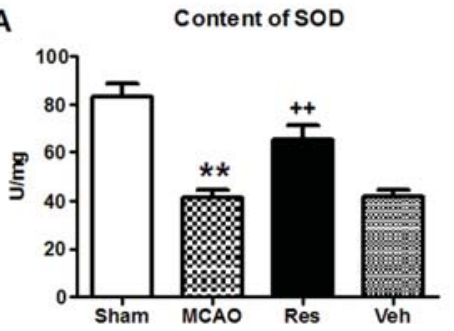

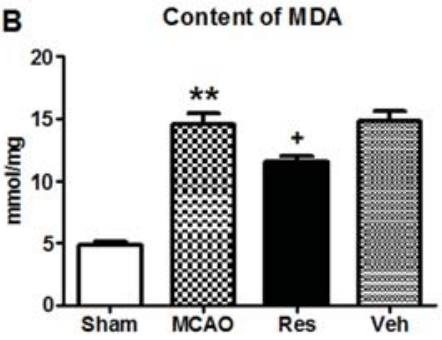

Fig. 3. Antioxidant effect of resveratrol in brain after $24 \mathrm{~h}$ of MCAO. (A) SOD activity in the ischemic tissue decreased $24 \mathrm{~h}$ after MCAO, and resveratrol significantly up-regulated SOD levels compared with the MCAO group ( ${ }^{* *} \mathrm{P}<0.01$ vs. Sham; $+\mathrm{P}<0.05,++\mathrm{P}<0.01$ vs. MCAO; $\mathrm{n}=10$ rats/group). (B) MDA content in ischemic tissue increased $24 \mathrm{~h}$ after MCAO, and resveratrol significantly reduced MDA levels compared with the MCAO group (**P $<0.01$ vs. Sham; $+\mathrm{P}<0.05,++\mathrm{P}<0.01$ vs. MCAO; $\mathrm{n}=10$ rats/group).

\section{Resveratrol protected against oxidative damage}

SOD is one of the most important antioxidases. MDA is a product of lipid peroxidation and is an indicator of oxidative stress. In this study, we used SOD and MDA as antioxidant markers. The tissue SOD antioxidant activity in the sham group was $82.88 \pm 19.04 \mathrm{U} / \mathrm{mg}$ protein. MCAO significantly decreased SOD activity $(41.42 \pm 9.58 \mathrm{U} / \mathrm{mg}, \mathrm{P}<0.01)$. Resveratrol significantly increased SOD activity $(65.09 \pm 18.93 \mathrm{U} / \mathrm{mg}, \mathrm{P}<0.01)$ compared with the MCAO group (Fig. 3A). As shown in Fig. 3B, the MDA levels increased significantly in the MCAO group, and resveratrol protected against oxidative damage by decreasing MDA levels. These data suggested that resveratrol was an effective antioxidant under cerebral ischemic conditions. 


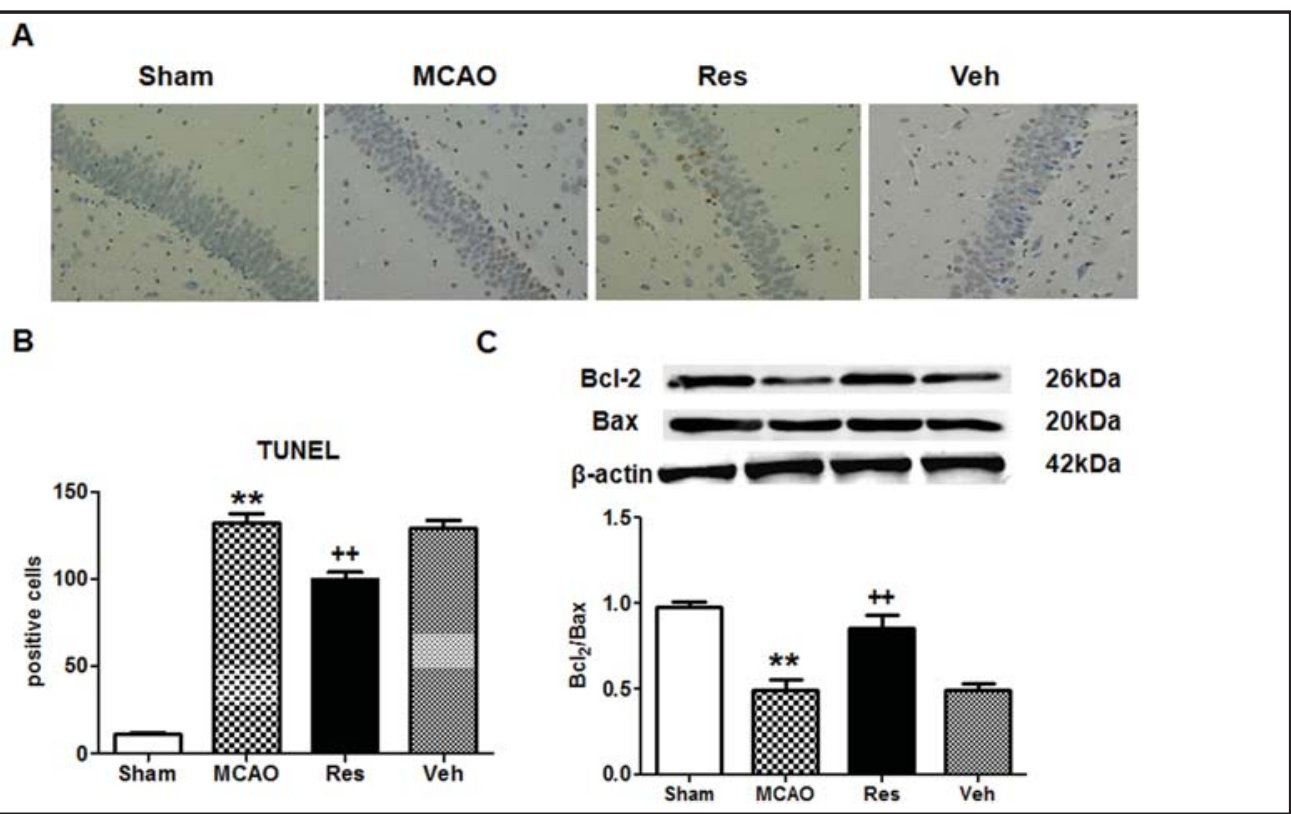

Fig. 4. Resveratrol reduced neurocyte apoptosis as evidenced by TUNEL staining and Western blot analysis after $24 \mathrm{~h}$ of cerebral ischemia. (A) TUNEL-positive staining was localized in the nuclei of neurons (brown in the nuclear area) as shown in the MCAO group. Fewer TUNEL-positive cells were observed in resveratroltreated rats (400X magnification). (B) Resveratrol pretreatment before cerebral ischemia markedly reduced the number of TUNEL-positive cells compared with the MCAO group ${ }^{* *} \mathrm{P}<0.01$ vs. Sham; $++\mathrm{P}<0.01$ vs. MCAO; $n=3$ rats/group, 3 section per group). (C) The Bcl-2/Bax ratio was significantly lower in the MCAO group compared with the sham group, and resveratrol significantly up-regulated the Bcl-2/Bax ratio $\left({ }^{* *} \mathrm{P}<\right.$ 0.01 vs. Sham; $++\mathrm{P}<0.01$ vs. MCAO; $\mathrm{n}=6$ rats/group).

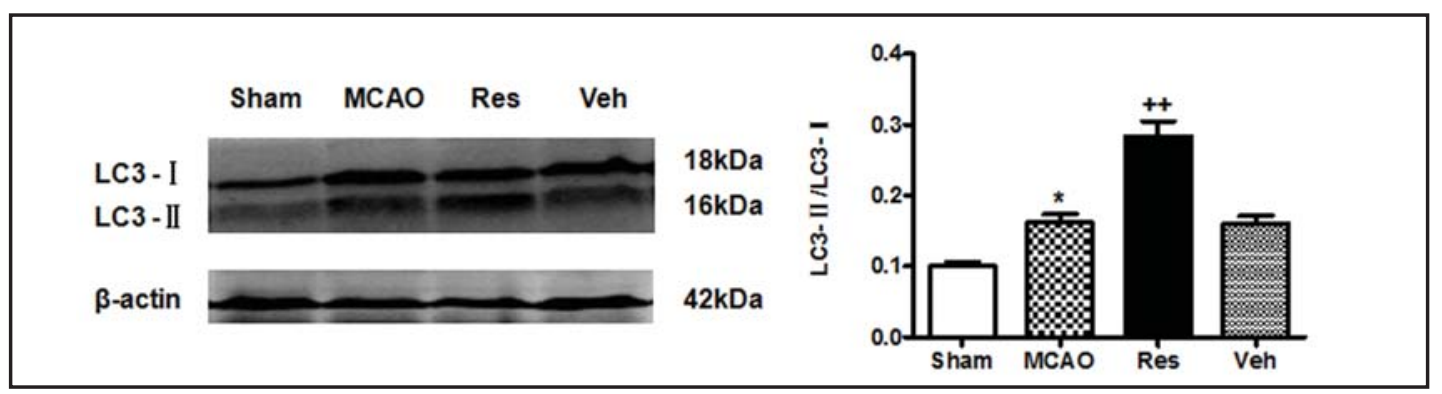

Fig. 5. Resveratrol increased the expression of the autophagy marker LC3 as determined by Western blotting. The levels of LC3-II/LC3-I in ischemic hippocampal tissue significantly increased after $24 \mathrm{~h}$ of cerebral ischemia, and resveratrol further increased the levels of LC3-II/LC3-I ${ }^{*} \mathrm{P}<0.05$ vs. Sham; $++\mathrm{P}<0.01$ vs. MCAO; $\mathrm{n}=6$ rats/group).

\section{Resveratrol prevented apoptosis after $24 \mathrm{~h}$ of cerebral ischemia}

Neurocyte death plays a central role in cerebral ischemia. To investigate the effect of resveratrol on cerebral ischemic injury, apoptosis was detected by TUNEL staining after ischemic brain injury. Cellular apoptosis significantly decreased in MCAO-injured animals pretreated with resveratrol (Fig. 4A). The number of TUNEL-positive cells was 130 cells per section in the infarct region of vehicle-treated animals, whereas this number was significantly reduced in the resveratrol-treated animals (98 cells per section) (Fig. 4B).

To further ascertain the role of resveratrol in neurocyte apoptosis after ischemic brain injury, Western blotting was performed to determine the relative levels of Bcl- 2 and Bax in the brain tissues from animals in the different groups (Fig. 4C). The anti-apoptotic protein Bcl-2 


\section{Cellular Physiology and Biochemistry}

Cell Physiol Biochem 2014;34:854-864

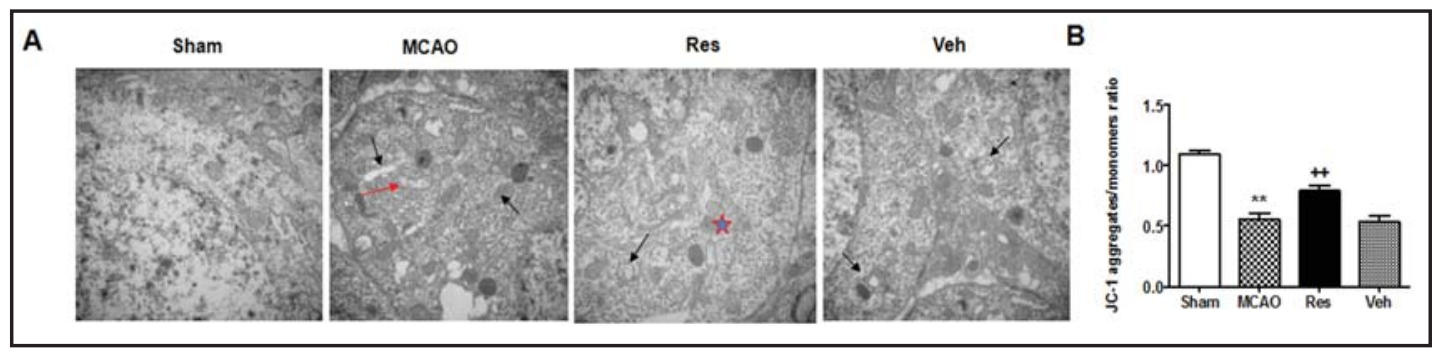

Fig. 6. Resveratrol protected mitochondria in hippocampal neurons. (A) Representative transmission electron microscopy images of hippocampal tissue. The arrows indicate normal mitochondria, the triangles indicate abnormal mitochondrial swelling, and the stars indicate autophagosome-containing mitochondria (2500X magnification). (B) Mitochondrial membrane potential in rat hippocampal neurons. Compared with the MCAO group, resveratrol increased the ratio of JC- 1 aggregates to JC- 1 monomers ${ }^{* *} \mathrm{P}<0.01$ vs. Sham; $++\mathrm{P}<0.01$ vs. MCAO; $\mathrm{n}=6$ rats/group).

Table 1. Effect of resveratrol on hemodynamic parameters of the heart in cerebral ischemia rats. LVSP: left ventricular systolic pressure; LVEDP: left ventricular end diastolic pressure; and $\pm \mathrm{dP} / \mathrm{dt}$ max: time derivatives of pressure. The data are presented as the mean \pm SEM $(* * \mathrm{P}$ $<0.01$ vs. Sham; $+\mathrm{P}<0.05$ vs. MCAO; $++\mathrm{P}<0.01$ vs. MCAO; $\mathrm{n}=8$ rats / group)

\begin{tabular}{ccccc}
\hline Group & LVSP & LVEDP & $+\mathrm{dP} / \mathrm{dt}$ & $-\mathrm{dP} / \mathrm{dt}$ \\
\hline & $(\mathrm{mmHg})$ & $(\mathrm{mmHg})$ & $(\mathrm{mmHg} / \mathrm{s})$ & $(\mathrm{mmHg} / \mathrm{s})$ \\
Sham & $90.7 \pm 3.8$ & $2.7 \pm 0.6$ & $4050 \pm 201$ & $3744 \pm 159$ \\
MCAO & $53.7 \pm 3.7^{* *}$ & $14.8 \pm 2.3^{* *}$ & $1432 \pm 202^{* *}$ & $1390 \pm 64^{* *}$ \\
Res & $63.5 \pm 3.6^{++}$ & $11 \pm 2.5^{+}$ & $1745 \pm 128^{+}$ & $1635 \pm 113^{+}$ \\
Veh & $52.5 \pm 5.2$ & $15.3 \pm 2.2$ & $1438 \pm 197$ & $1388 \pm 61$ \\
\hline
\end{tabular}

and the pro-apoptotic protein Bax are crucial determinants of the apoptotic response. Our data revealed that compared with the MCAO group, the ratio of Bcl-2/Bax in the resveratrol group was increased, and there was no obvious change in the vehicle control group. These data indicated that resveratrol significantly alleviated neuronal apoptosis by up-regulating the Bcl-2/Bax ratio after ischemia-induced brain injury.

\section{Resveratrol increased autophagy after 24 h of cerebral ischemia}

LC3-II is required for the formation of autophagosomes and is a biomarker of autophagosomes in mammalian cells. LC3-II is the cleaved and lipidated form of cytosolic LC3-I. The protein levels of LC3 were determined by Western blotting. Our data indicated an increase in the LC3-II/LC3-I ratio in the MCAO group compared with the sham group. Resveratrol further increased this ratio compared with the MCAO group (Fig. 5). These data indicated that resveratrol might enhance neuronal autophagy by increasing LC3II expression.

\section{Resveratrol improved the mitochondrial integrity in neurons}

Transmission electron microscopy was performed on samples from each group $24 \mathrm{~h}$ after cerebral ischemia. The hippocampal structure in the sham group was normal as indicated by the number of mitochondria and the intact appearance of the mitochondrial organelles (Fig. 6A). Compared with the sham group, most of the mitochondria from the MCAO group suffered severe ischemic damage with swollen and fractured structures. In the resveratrol pretreatment group, only a few mitochondria were swollen and a few autophagosomes were observed, but the vehicle group exhibited no protective effect against ischemic damage. These data implied that resveratrol pretreatment protected mitochondria from structural damage in response to cerebral ischemia. 
A

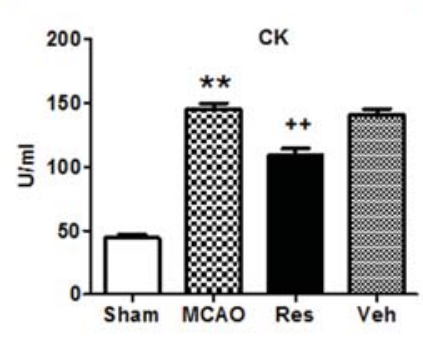

B

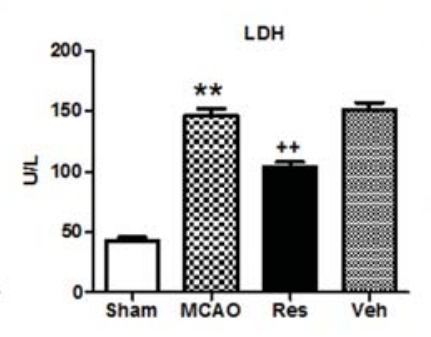

D

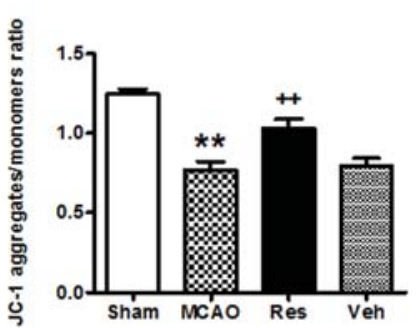

C

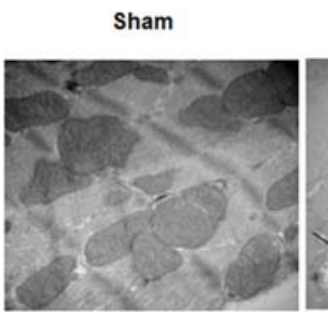

MCAO

Res

Veh
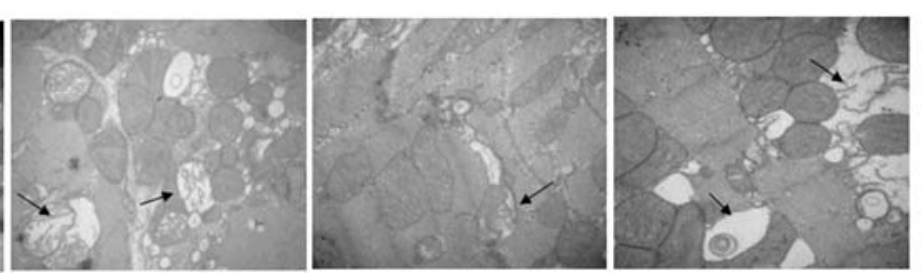

Fig. 7. Resveratrol prevented cardiomyocyte damage induced by cerebral ischemia. (A) Resveratrol downregulated CK levels after $24 \mathrm{~h}$ of MCAO (**P $<0.01$ vs. Sham; $++\mathrm{P}<0.01$ vs. MCAO; $\mathrm{n}=10$ rats/group). (B) Resveratrol down-regulated LDH levels after $24 \mathrm{~h}$ of MCAO (**P $<0.01$ vs. Sham; ++P $<0.01$ vs. MCAO; $=10$ rats/group). (C) Representative transmission electron microscopy images illustrate severely damaged myocardium in cerebral ischemic rats. The arrows indicate normal mitochondria, and the triangles indicate abnormal mitochondrial swelling (2500X magnification). (D) Mitochondrial membrane potential in the left ventricle. Resveratrol pretreatment decreased the depolarization of mitochondria in the myocardium of cerebral ischemic rats ( $* * \mathrm{P}<0.01$ vs. Sham $++\mathrm{P}<0.01$ vs. MCAO; $\mathrm{n}=6$ rats/group).

To further illustrate the protective effect of resveratrol regarding mitochondrial damage, $\Delta \psi \mathrm{m}$ was measured using JC- 1 staining. A decrease in $\Delta \psi \mathrm{m}$ indicates mitochondrial damage. Cerebral ischemia in the MCAO group significantly reduced the neuronal $\Delta \psi \mathrm{m}$, as indicated by a decrease in the ratio of JC- 1 aggregates to JC-1 monomers (Fig. 6B). Compared with the MCAO group, the resveratrol pretreatment group exhibited an increased ratio of JC-1 aggregates to JC- 1 monomers, suggesting that fewer neuronal mitochondria were depolarized. In the sham group, JC-1 existed in the aggregate form.

\section{Cardioprotective effect of resveratrol against ischemia-induced brain injury}

A growing number of studies have suggested that one of the important causes of the high mortality from strokes is cardiac dysfunction induced by cerebral ischemia. To investigate the effect of resveratrol on cardiac function after $24 \mathrm{~h}$ of MCAO, hemodynamic parameters were measured in the four groups (Table 1). The LVEDP was significantly increased, $+\mathrm{dP} /$ $\mathrm{dt}$ and $-\mathrm{dP} / \mathrm{dt}$ were both significantly decreased, and the LVSP was decreased in the MCAO group compared with the sham group. These data suggested that both the diastolic and systolic functions of the heart were severally impaired after cerebral ischemia. Resveratrol pretreatment significantly changed the LVEDP, $+\mathrm{dP} / \mathrm{dt}$ and $-\mathrm{dP} / \mathrm{dt}$ as well as the LVSP compared with the MCAO group, but the vehicle group did not show any improvement in cardiac function. The serum levels of the diagnostic marker enzymes, LDH and CK, were analyzed. As shown in Fig. 7A and Fig. 7B, the levels of both markers were significantly higher in the MCAO group than in the sham group. Pretreatment with resveratrol significantly decreased the levels of CK and LDH compared with the MCAO group.

Transmission electron microscopy was also performed on cardiac myocytes to further determine the role of resveratrol. The mitochondria in the MCAO group were swollen and exhibited significant disruptions or loss of cristae compared with the sham group. Myofibrils 


\section{Cellular Physiology and Biochemistry}

Cell Physiol Biochem 2014;34:854-864

\begin{tabular}{l|l}
\hline DOI: $10.1159 / 000366304$ & (C) 2014 S. Karger AG, Basel
\end{tabular}

Wang et al.: Protective Roles of Resveratrol in Cerebral Ischemia

were arranged irregularly. In the resveratrol-pretreated group, certain nuclei exhibited pathological changes, but these were relatively mild, and most of the mitochondria were intact. In the vehicle group, the mitochondria suffered severe damage (Fig. 7C). The $\Delta \psi \mathrm{m}$ data in the cardiomyocytes further illustrated the protective effect of resveratrol in mitochondria. As shown in Fig. 7D, $\Delta \psi \mathrm{m}$ decreased significantly in the MCAO group compared with the sham group. However, the $\Delta \psi \mathrm{m}$ was dramatically increased in the resveratrol pretreatment group compared with the MCAO group, providing direct evidence that resveratrol protected mitochondrial function during cerebral ischemia. These data suggested that resveratrol significantly attenuated myocardial damage induced by cerebral ischemia.

\section{Discussion}

In the present study, we found that pretreatment with resveratrol effectively alleviated the neuronal and myocardial damage following MCAO. Pretreatment with resveratrol (30 $\mathrm{mg} / \mathrm{kg} / \mathrm{d}$ for 7 days) significantly reduced mortality, neurological scores and infarct volume, rescued mitochondria, improved cellular autophagy by increasing LC3II protein expression, prevented cellular apoptosis by increasing the Bcl-2/Bax ratio, and exerted anti-oxidant effects by increasing the activity of the antioxidant enzyme SOD as well as decreasing the formation of the lipid peroxidation product MDA after cerebral ischemia. Our data demonstrated that resveratrol significantly induced autophagy in the rat stroke model and attenuated myocardial injury that accompanies cerebral ischemia.

Mitochondria are the primary intracellular source of ROS and play a central role in the exacerbation of cerebral ischemia injury [28]. ROS levels are regulated by endogenous antioxidants and antioxidases, such as GSH, SOD, CAT, bilirubin and Coenzyme Q. SOD is a particularly important antioxidase, and generally its activity indirectly reflects the body's ability to clear ROS. In response to oxidative stress, MDA is a poisonous end-product of lipid peroxidation, and its levels directly reflect the rate and extent of lipid peroxidation. In the present study, resveratrol treatment before cerebral ischemia injury restored SOD activity and decreased MDA levels compared with the MCAO group, suggesting that the antioxidant effect of resveratrol may be achieved by protecting the mitochondria; however, additional studies are necessary to confirm this hypothesis.

Although the mechanisms of cell death after cerebral ischemia remain unclear, mitochondria play an important role by activating signaling pathways via ROS production or by regulating mitochondria-dependent apoptosis pathways. It is well known that the balance between anti-apoptotic Bcl-2 and pro-apoptotic Bax plays an important role in regulating apoptosis. In agreement with previous studies [29], our data verified that resveratrol significantly improved the Bcl-2/Bax ratio after cerebral ischemia. This evidence strongly supported our hypothesis that resveratrol confers neuroprotection by targeting mitochondria and inhibiting apoptosis.

Apoptotic and autophagic mechanisms can be activated in the same injured neuron, and autophagy may inhibit apoptosis by activating cytoprotective pathways [30,31]. Mitochondria are both a major source of and a target of ROS. Excess ROS can elicit the oxidative modification of mitochondrial proteins, lipids, and mtDNA, resulting in mitochondrial dysfunction. During stress, autophagy is an self-protective response that promotes survival by removing damaged mitochondria [11]. To explore possible ways that resveratrol protects the brain during ischemia, we analyzed LC3-II protein expression as a marker of autophagy. The results of this study indicated that resveratrol pretreatment resulted in increased LC3-II formation compared with the MCAO and vehicle groups. These data suggested that mitochondria are involved in cerebral ischemic damage via interactions with anti-apoptotic Bcl-2 family members and autophagy. The transmission electron microscopy data corresponded with these results, clearly demonstrating that the normal structure was disrupted after ischemia, as indicated by the disorganization of neurons, the swelling of mitochondria and the formation of autophagosomes. Resveratrol treatment elicited autophagy as indicated by the 


\section{Cellular Physiology and Biochemistry}

Cell Physiol Biochem 2014;34:854-864

\begin{tabular}{l|l}
\hline DOI: $10.1159 / 000366304$ & (c) 2014 S. Karger AG, Basel
\end{tabular}

www.karger.com/cpb

Wang et al.: Protective Roles of Resveratrol in Cerebral Ischemia

presence of autophagosomes containing mitochondria enclosed in a double membrane (the star indicates an autophagosome in Fig. 6A).

Our previous study characterized cerebral ischemia-induced cardiovascular disturbances by demonstrating significant myocardial damage using transmission electron microscopy [26]. CK and LDH are sensitive indices for evaluating the severity of myocardial damage. In the present study, resveratrol significantly down-regulated the excessive MCAOinduced release of CK and LDH in the serum. Interestingly, pretreatment with resveratrol also attenuated myocardial mitochondrial structural damage and the mitochondrial membrane potential decline after $24 \mathrm{~h}$ of MCAO (Fig. 7C and Fig. 7D). This finding further indicated that resveratrol might be involved in protecting mitochondria during myocardial damage induced by MCAO. Hemodynamic parameters were also measured in the four groups of rats. Our observations suggested that resveratrol pretreatment attenuated cardiac contractile dysfunction in cerebral ischemic rats, which was supported by the increase in LVSP and $\pm \mathrm{dP}$ / $\mathrm{dt}$ and the decrease in LVEDP (Table 1).

In summary, resveratrol is a unique compound that protected against neurological and related cardiovascular damage induced by experimental stroke through multiple mechanisms, including decreasing oxidative damage and apoptosis and regulating mitochondria. These data suggested that resveratrol is a promising neuroprotective and cardioprotective therapeutic for cerebral ischemia. However, the detailed molecular interactions between resveratrol and mitochondrial proteins remain to be elucidated, and identifying these interactions will be beneficial for understanding the mechanisms of resveratrol-mediated neuroprotection and mitochondria-targeted therapeutics against stroke and related myocardial injury.

\section{References}

1 Strong K, Mathers C, Bonita R: Preventing stroke: Saving lives around the world. Lancet Neurol 2007;6:182187.

- Butcher KS, Parsons MW: Cardiac enzyme elevations after stroke: The importance of specificity. Stroke 2002;33:1944-1945; author reply 1944-1945.

-3 Oppenheimer SM, Hachinski VC: The cardiac consequences of stroke. Neurol Clin 1992;10:167-176.

$\checkmark 4$ Amaro S, Llull L, Urra X, Obach V, Cervera A, Chamorro A: Risks and benefits of early antithrombotic therapy after thrombolytic treatment in patients with acute stroke. PLoS One 2013;8:e71132.

5 Wechsler LR: Intravenous thrombolytic therapy for acute ischemic stroke. N Engl J Med 2011;364:21382146.

-6 Greenlund LJ, Deckwerth TL, Johnson EM, Jr:: Superoxide dismutase delays neuronal apoptosis: A role for reactive oxygen species in programmed neuronal death. Neuron 1995;14:303-315.

7 Firuzi 0, Miri R, Tavakkoli M, Saso L: Antioxidant therapy: Current status and future prospects. Curr Med Chem 2011;18:3871-3888.

$>8$ Antonawich FJ, Krajewski S, Reed JC, Davis JN: Bcl-x(l) bax interaction after transient global ischemia. J Cereb Blood Flow Metab 1998;18:882-886.

-9 Ouyang YB, Giffard RG: Cellular neuroprotective mechanisms in cerebral ischemia: Bcl-2 family proteins and protection of mitochondrial function. Cell Calcium 2004;36:303-311.

10 Levine B, Klionsky DJ: Development by self-digestion: Molecular mechanisms and biological functions of autophagy. Dev Cell 2004;6:463-477.

11 Kubli DA, Gustafsson AB: Mitochondria and mitophagy: The yin and yang of cell death control. Circ Res 2012;111:1208-1221.

12 Ohshiro K, Rayala SK, El-Naggar AK, Kumar R: Delivery of cytoplasmic proteins to autophagosomes. Autophagy 2008;4:104-106.

13 Tang Q, Li G, Wei X, Zhang J, Chiu JF, Hasenmayer D, Zhang D, Zhang H: Resveratrol-induced apoptosis is enhanced by inhibition of autophagy in esophageal squamous cell carcinoma. Cancer Lett 2013;336:325337. 


\section{Cellular Physiology and Biochemistry}

Cell Physiol Biochem 2014;34:854-864

\begin{tabular}{l|l}
\hline DOI: $10.1159 / 000366304$ & (c) 2014 S. Karger AG, Basel
\end{tabular}

Wang et al.: Protective Roles of Resveratrol in Cerebral Ischemia

14 Renaud S, de Lorgeril M: Wine, alcohol, platelets, and the french paradox for coronary heart disease. Lancet 1992;339:1523-1526.

15 Sinha K, Chaudhary G, Gupta YK: Protective effect of resveratrol against oxidative stress in middle cerebral artery occlusion model of stroke in rats. Life Sci 2002;71:655-665.

16 Patel KR, Scott E, Brown VA, Gescher AJ, Steward WP, Brown K: Clinical trials of resveratrol. Ann N Y Acad Sci 2011;1215:161-169.

17 Lin MT, Beal MF: Mitochondrial dysfunction and oxidative stress in neurodegenerative diseases. Nature 2006;443:787-795.

18 Vosler PS, Graham SH, Wechsler LR, Chen J: Mitochondrial targets for stroke: Focusing basic science research toward development of clinically translatable therapeutics. Stroke 2009;40:3149-3155.

19 Rimbaud S, Ruiz M, Piquereau J, Mateo P, Fortin D, Veksler V, Garnier A, Ventura-Clapier R: Resveratrol improves survival, hemodynamics and energetics in a rat model of hypertension leading to heart failure. PLoS One 2011;6:e26391.

-20 Dasgupta B, Milbrandt J: Resveratrol stimulates amp kinase activity in neurons. Proc Natl Acad Sci U S A 2007;104:7217-7222.

-21 Cadenas S, Barja G: Resveratrol, melatonin, vitamin e, and pbn protect against renal oxidative DNA damage induced by the kidney carcinogen kbro3. Free Radic Biol Med 1999;26:1531-1537.

22 Wang Q Xu J, Rottinghaus GE, Simonyi A, Lubahn D, Sun GY, Sun AY: Resveratrol protects against global cerebral ischemic injury in gerbils. Brain Res 2002;958:439-447.

-23 Aspey BS, Taylor FL, Terruli M, Harrison MJ: Temporary middle cerebral artery occlusion in the rat: Consistent protocol for a model of stroke and reperfusion. Neuropathol Appl Neurobiol 2000;26:232-242.

-24 Yang B, Lin H, Xu C, Liu Y, Wang H, Han H, Wang Z: Choline produces cytoprotective effects against ischemic myocardial injuries: Evidence for the role of cardiac m3 subtype muscarinic acetylcholine receptors. Cell Physiol Biochem 2005;16:163-174.

25 Aspey BS, Cohen S, Patel Y, Terruli M, Harrison MJ: Middle cerebral artery occlusion in the rat: Consistent protocol for a model of stroke. Neuropathol Appl Neurobiol 1998;24:487-497.

-26 Sun L, Du J, Zhang G, Zhang Y, Pan G, Wang L, Yang B: Aberration of l-type calcium channel in cardiac myocytes is one of the mechanisms of arrhythmia induced by cerebral ischemia. Cell Physiol Biochem 2008;22:147-156.

27 Sun L, Ai J, Wang N, Zhang R, Li J, Zhang T, Wu W, Hang P, Lu Y, Yang B: Cerebral ischemia elicits aberration in myocardium contractile function and intracellular calcium handling. Cell Physiol Biochem 2010;26:421430.

28 Niizuma K, Endo H, Chan PH: Oxidative stress and mitochondrial dysfunction as determinants of ischemic neuronal death and survival. J Neurochem 2009;109 Suppl 1:133-138.

29 Xing B, Chen H, Zhang M, Zhao D, Jiang R, Liu X, Zhang S: Ischemic postconditioning inhibits apoptosis after focal cerebral ischemia/reperfusion injury in the rat. Stroke 2008;39:2362-2369.

-30 Rami A, Langhagen A, Steiger S: Focal cerebral ischemia induces upregulation of beclin 1 and autophagylike cell death. Neurobiol Dis 2008;29:132-141.

-31 Ginet V, Puyal J, Clarke PG, Truttmann AC: Enhancement of autophagic flux after neonatal cerebral hypoxiaischemia and its region-specific relationship to apoptotic mechanisms. Am J Pathol 2009;175:1962-1974. 